\title{
EFFECT OF SURFACE TREATMENT AND ARTIFICIAL AGING ON MICROTENSILE BOND STRENGTH OF ZIRCONIA TO RESIN CEMENT
}

\author{
Ahmed M. Hamdy* and Abou Bakr Hossam Hashem**
}

\begin{abstract}
Statement of problem: Reliable bond to zirconia based materials has proven to be difficult which is the major limitation against luting zirconia restorations.

Purpose: This in vitro study compared effect of selective infiltration etching (SIE) and tribochemical silica sandblasting (TSS) on microtensile bond strength to zirconia based material before and after different artificial aging intervals.

Materials and methods: Twenty Lava plus high translucency zirconia disks (3 M, ESPE) were randomly divided into two study groups according to their surface treatment: (a) selective infiltration etching (SIE) and (b) tribo-chemical silica sandblasting (TSS). Zirconia disks were bonded to composite resin specimens using Panavia F2.0 resin cement. Zirconia resin bond strength was evaluated using the microtensile bond strength test (MTBS) and the test was repeated before and after the following intervals of accelerated artificial aging; 1 week, 2 weeks, 3 weeks and 4 weeks of water storage $\left(37^{\circ} \mathrm{C}\right)$. A repeated measures ANOVA and Bonferroni post-hoc tests were used to analyze the data.
\end{abstract}

Results: The interaction between surface treatments and storage times were statistically significant. Data analysis revealed significant differences between the two surface treatments $(\mathrm{P}<0.05)$.Selective infiltration etched specimens demonstrated relatively higher MTBS values than TSS (48.5 and 24.8 MPa).SEM examination of broken microbars revealed more interfacial failure type for TSS than SIE specimens that showed a predominantly cohesive failure type.

Conclusions: Selective infiltration etching provided superior micro-tensile bond strength results compared to tribo-chemical silica sandblasting. Established bond strength by selective infiltration etching was not affected by artificial aging as tribo-chemical silica sandblasting.

\footnotetext{
* Associate Professor, Fixed Prosthodontic Department, Faculty of Dentistry, MSA University.

** Lecturer, Research Institute of Ophthalmology, Dental Department.
} 


\section{INTRODUCTION}

Zirconia based materials are dynamic on microscopic structural level. These materials can undergo transformational phase changes in response to thermal and mechanical stresses ${ }^{(1)}$. Structural changes occur on the grain level, which tends to mature and grow in size when given sufficient time and temperature. Grain growth and cubic grain formation where observed when zirconia was heated to $1450^{\circ} \mathrm{C}$ for 2 hours ${ }^{(2)}$. When heated for 30 minutes at lower temperatures $\left(700-900^{\circ} \mathrm{C}\right)$, the surface of zirconia was reported to undergo a thermal aging process, especially in the presence of water vapor. On a microscopic level, thermal aging resulted in the creation of surface elevations, grain pullout, detachment and increase grain boundary thickness ${ }^{(3,4)}$.

During thermal etching of zirconia at a temperature of $1350^{\circ} \mathrm{C}$ for 12 minutes, surface elevations, rippled grain surfaces and vertical grooves at grain boundaries were previously reported using electron microscope. These observations were related to the tetragonal monoclinic transformation of zirconia crystals at the surface grains, which can accommodate the accompanying increase in volume, a state determined by grain boundaries and surface energies ${ }^{(4,5)}$. However, deeper grains are restrained and constrained by the bulk of the materials and thus become strained ${ }^{(5)}$.

Several mechanical and chemical surface pretreatments have been recommended to increase the bonding effectiveness of composite cement to zirconia. Former pre-treatments such as surface grinding using diamond burs ${ }^{(6)}$, chemical etching using hydrofluoric $\operatorname{acid}^{(7)}$ and laser irradiation (8) were applied to roughen the surface of zirconia ceramics, but none of them resulted in durable bonding to zirconia ${ }^{(6)}$.

It appears essential to create both micromechanically prepared and a chemically activated surface. For instance, tribo-chemical silica sandblasting with 30 and $110 \mu \mathrm{m}$ silica-coated aluminum oxide particles roughens and also chemically activates zirconia, thus making it more receptive for chemically bonding via silane coupling agents $^{(8)}$.

Previous in vitro study ${ }^{(10)}$ showed that combination of mechanical pre-treatment using tribo-chemical silica sandblasting (Cojet, 3M ESPE; Seefeld, Germany) and chemical pre-treatment using a ceramic primer provided the highest bonding effectiveness to dental zirconia. More-over, the resultant bond appeared insensitive to mechanical $\operatorname{aging}^{(11)}$.The difference between conventional A12O3 sandblasting and tribo-chemical silica sandblasting in terms of their efficacy in making zirconia receptive to bonding is still unclear.

Tribo-chemical silica sandblasting with 30 and $110 \mu \mathrm{m}$ silica-coated aluminum oxide particles has been shown not only to roughen but also to chemically activate zirconia, thus making it more receptive for chemical bonding via silane coupling agents. To avoid the well-documented sub-surface damage and transformation induced by high pressure and big particle size, one should apply air abrasion at lower pressure(1-2 bars) using particles up to $50 \mu \mathrm{m}$ in $\operatorname{size}^{(2,3)}$. The sole application of traditional ceramic (silane) primers appeared not very effective on zirconia ${ }^{(4,6)}$, while the application of 10-MDP containing primers has been documented to chemically bond to zirconia, especially when applied on previously air -abraded zirconia using 50 to $110 \mu \mathrm{m}$ alumina particles or $110 \mu \mathrm{m}$ silica coated alumina sand ${ }^{(14,15)}$.

This study aimed to assess the effect of two surface pre-treatments on micro-tensile bond strength to translucent zirconia after subjecting specimens to artificial aging. The null hypothesis tested were (1) bonding efficacy of resin-composite cement to zirconia was not different for the two pretreatments tested, and (2) the strength of the tested samples was not affected by artificial aging. 


\section{MATERIALS AND METHODS}

Twenty Lava plus highly translucent zirconia disks (3M, ESPE), (diameter :19.5 mm; thickness: 3 $\mathrm{mm}$ ) were prepared as described in previous study ${ }^{(16)}$, cutting procedure was precisely guided and carried out with a horizontally moving digital micrometer (IDC 1508, mitutoyo; Kawasaki, Japan).

Samples were polished using silicon carbide papers starting with a 120 grit and ending with a 800 grit (Microcut, Buehler). Polishing was carried out by using a rotating metallographic polishing device (Ecomet, Buehler) under a $300 \mathrm{gm}$ load and water cooling .

10 disks (group 1) underwent selective infiltration etching (SIE) surface treatment ${ }^{(16)}$, the method employs a heat induced maturation process to pre-stress surface grain boundaries on zirconia to allow infiltration of molten glass. Zirconia is heated to $750^{\circ} \mathrm{C}$ for 2 minutes, cooled to $650^{\circ} \mathrm{C}$ for 1 minute, reheated to $750^{\circ} \mathrm{C}$ for an additional 1 minute and then cooled to room temperature .After this heat treatment, the grain boundaries become pre-stressed and can be easily infiltrated by other materials. After cooling to room temperature, the glass is then etched using 5\% hydrofluoric acid for $30 \mathrm{~min}$. this creates rough surface topography with deep grooves at zirconia grain boundaries, allowing nanomechanical interlocking of resin composite cement.

10 disks (group 2) underwent tribo-chemical silica sandblasting (Co Jet, 3M ESPE; Seefeld, Germany).

A total of 20 resin composite disks (Filtek Z 250, 3M, ESPE; St Paul, MN, USA) measuring $18.0 \mathrm{~mm}$ in diameter and $3 \mathrm{~mm}$ in thickness were prepared by injecting the composite resin into a mold and light polymerized for $20 \mathrm{sec}$ each from the top and bottom (Elipar Free Light 2,3 M ESPE).

All Disks were coated with a freshly mixed resin composite cement (Panavia F 2.0; Kuraray Co. Ltd.,
Tokyo, Japan) and seated on top of the surface of the treated zirconia specimens under a fixed load of $20 \mathrm{~N}$. Excess cement was wiped off and an air barrier gel was applied as a coating around the margins of the double-layered specimens.

After storage at room temperature for $24 \mathrm{~h}$, the specimens were cut into 10 microbars per group ( $9 \mathrm{~mm}$ length, $2 \mathrm{~mm} \times 2 \mathrm{~mm}$ in cross-section) using a precision cutting device (Isomet 1000; Buehler, Lake Bluff, IL) and a diamond-coated saw (Diamond Wafering Blade, No. 11-4276; Buehler) under water cooling ${ }^{(14)}$. The microbars were glued to the attachment unit (Clearfil SE Bond, Kuraray) taking care to center the resin-to-zirconia interface in the free space of the attachment unit. Zirconiaresin micro-tensile bond strength was measured by applying an axial load on the bonded interface using a universal testing machine (Instron 6022; Instron Corp., High Wycombe, England).

The load cell $(100 \mathrm{~N})$ was calibrated using standardized loads and the cross-head speed was calibrated using a digital micrometer (Millitron; Feinpruf Perthen, Gottingen, Germany).

Tests were conducted to the 10 test groups, immediately, then a week, two weeks, three weeks and four weeks from cementation time, during which time the microbars were stored in demineralized water at $37^{\circ} \mathrm{C}$ (T 340; Heraeus Kulzer). Failure load was extracted from computer generated data files. The broken microbars were ultrasonically cleaned, dried, gold sputter coated, and examined under a SEM (XL20; Philips, Eindhoven, the Netherlands) and failure type was classified into interfacial failure across the zirconia-resin interface resulting in exposure of the zirconia surface or cohesive failure in the composite material or the adhesive resin (Fig.2-3).

A repeated measure analysis of variance (ANOVA) was selected to analyze the data with 1 factor (time, 5 levels). Bonferroni post hoc test was selected for pairwise comparisons $(\alpha=.05)$. 
The sample size ( $\mathrm{n}=10$ discs/group) was based on a power analysis (power=1) set to detect medium effect size differences $(\mathrm{F}=0.25)$ which, in terms of the bonding technique and MTBS values, could be of clinical relevance (Sigma-Stat Version 3.0; SPSS Inc, Chicago, Ill). One-way analysis of variance was used to analyze the data.

\section{RESULTS}

Data analysis table 1, graph. 1 revealed significant differences between the two surface treatments $(\mathrm{P} \leq 0.05)$. On the other hand, SIE specimens demonstrated relatively higher MTBS

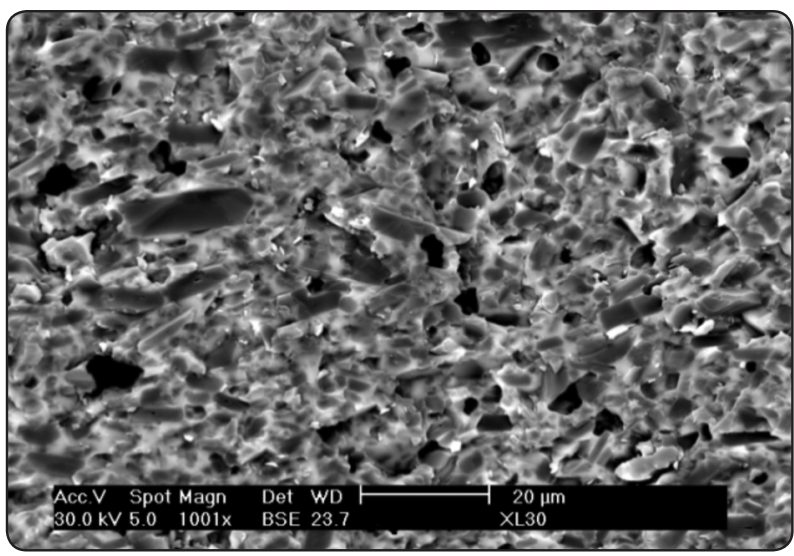

Fig. (1) SEM image after SIE of Zirconia x 1000

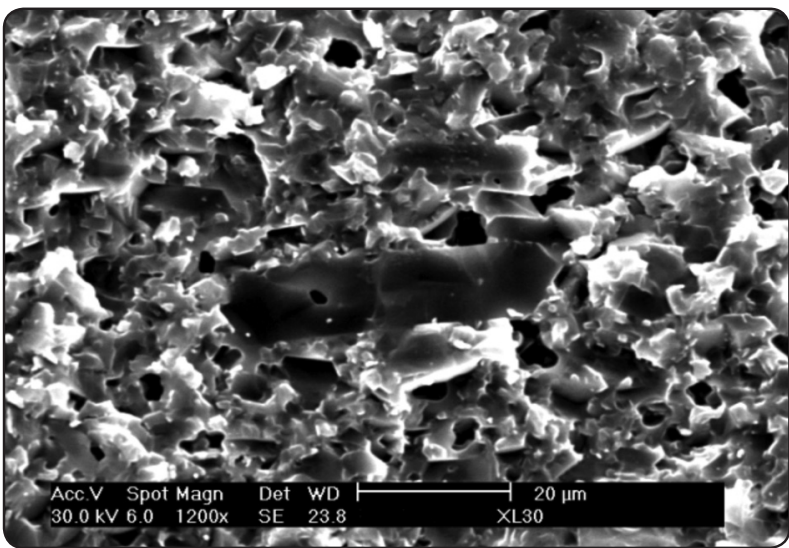

Fig. (3) SEM image showing interfacial failure of TSS specimen $\mathrm{x} 1200$ values than TSS (48.5 and 24.8 MPa).

In table 2 , graph. 2 there was a significant interaction between water storage time and the bonding technique $\mathrm{P}<0.001$ for TSS groups. MTBS values were stable overall of testing intervals for SIE groups.

SEM examination of broken microbars revealed more interfacial failure type for TSS specimens than SIE specimens that showed a predominantly cohesive failure type in resin cement as shown in fig. 2,4 .

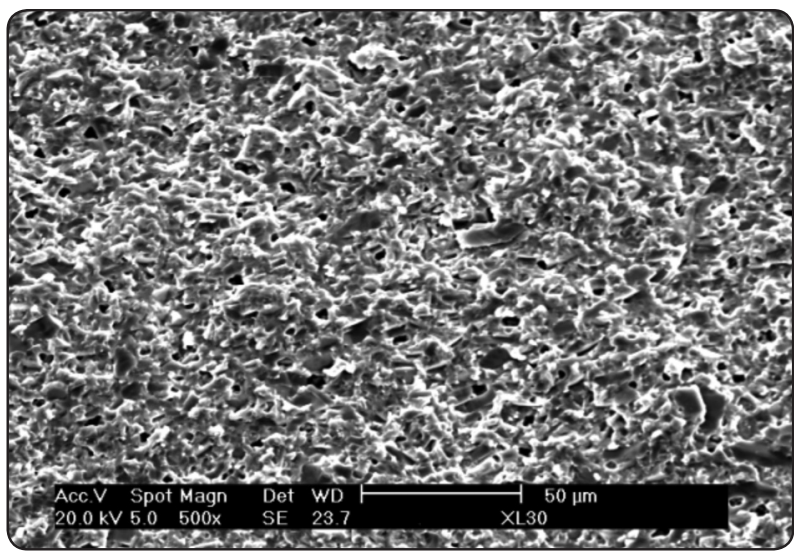

Fig. (2) SEM image showing cohesive failure in resin cement $\mathrm{x} 500$.

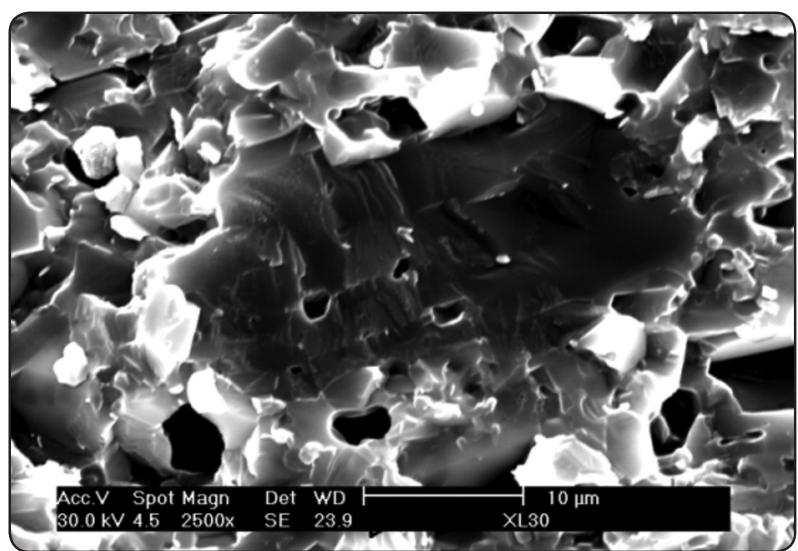

Fig. (4) SEM image showing microgaps due to hydrolysis of TSS specimen $\mathrm{x} 2500$. 
TABLE (1) Microtensile bond strength (Mpa) of groups at different time intervals

\begin{tabular}{|c|c|c|c|c|c|c|c|c|c|c|c|}
\hline \multirow[t]{2}{*}{ Groups } & \multicolumn{2}{|c|}{ Zero time } & \multicolumn{2}{|c|}{ Week 1} & \multicolumn{2}{|c|}{ Week 2} & \multicolumn{2}{|c|}{ Week 3} & \multicolumn{2}{|c|}{ Week 4} & \multirow{2}{*}{$\begin{array}{c}\text { P value } * \\
\text { for time } \\
\text { effect }\end{array}$} \\
\hline & Mean & (SD) & Mean & $(\mathrm{SD})$ & Mean & $(\mathrm{SD})$ & Mean & $(\mathrm{SD})$ & Mean & (SD) & \\
\hline SIE & 48.50 & $(1.72)$ & 46.90 & (1.66) & 47.80 & (1.99) & 47.60 & (1.78) & 45.90 & $(1.72)$ & \\
\hline TSS & $28.40 \mathrm{~A}$ & (2.17) & $29.40 \mathrm{~A}$ & $(0.84)$ & $27.40 \mathbf{A B}$ & (2.07) & $28.10 \mathrm{~A}$ & $(2.02)$ & $24.90 \mathbf{B}$ & $(2.17)$ & \\
\hline
\end{tabular}

* P value is significant $£ 0.05$, All comparisons are Bonferroni adjusted. Time points sharing same letter are not significantly different

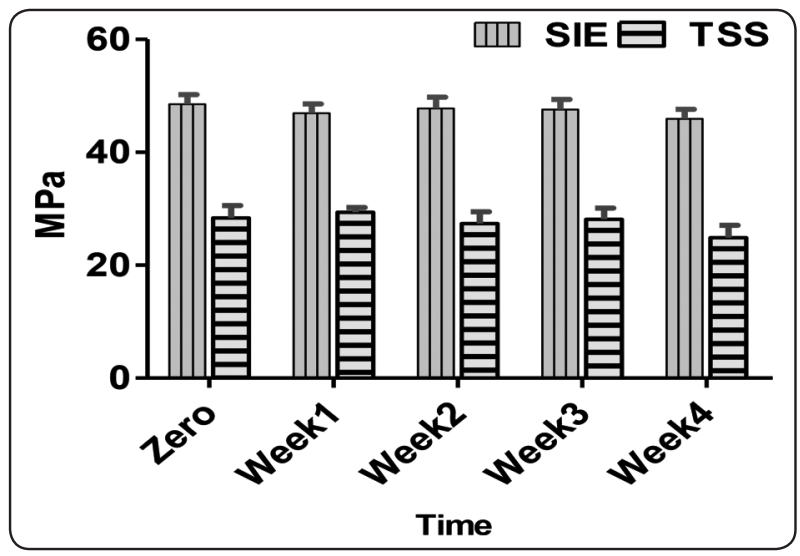

Graph. (1) Mean MTBS at different time intervals.

TABLE (2) Different types and percentage of failures.

\begin{tabular}{|c|c|c|c|c|c|c|}
\hline & \multicolumn{4}{|c|}{ Study group } & \multirow{3}{*}{$\mathrm{P}$ value } \\
\hline & & \multicolumn{2}{|c|}{ SIE } & \multicolumn{2}{|c|}{ TSS } & \\
\hline & & No & $\%$ & No & $\%$ & \\
\hline \multirow{3}{*}{ Mode of failure (0) } & Cohesive & 10 & 100.0 & 6 & 60.0 & \multirow{3}{*}{0.09} \\
\hline & Interfacial & 0 & 0.0 & 4 & 40.0 & \\
\hline & Total & 10 & 100.0 & 10 & 100.0 & \\
\hline \multirow{3}{*}{ Mode of failure (w1) } & Cohesive & 9 & 90.0 & 7 & 70.0 & \multirow{3}{*}{0.58} \\
\hline & Interfacial & 1 & 10.0 & 3 & 30.0 & \\
\hline & Total & 10 & 100.0 & 10 & 100.0 & \\
\hline \multirow{3}{*}{ Mode of failure (w2) } & Cohesive & 10 & 100.0 & 7 & 70.0 & \multirow{3}{*}{0.21} \\
\hline & Interfacial & 0 & 0.0 & 3 & 30.0 & \\
\hline & Total & 10 & 100.0 & 10 & 100.0 & \\
\hline \multirow{3}{*}{ Mode of failure (w3) } & Cohesive & 8 & 80.0 & 5 & 50.0 & \multirow{3}{*}{0.35} \\
\hline & Interfacial & 2 & 20.0 & 5 & 50.0 & \\
\hline & Total & 10 & 100.0 & 10 & 100.0 & \\
\hline \multirow{3}{*}{ Mode of failure (w4) } & Cohesive & 9 & 90.0 & 6 & 60.0 & \multirow{3}{*}{0.30} \\
\hline & Interfacial & 1 & 10.0 & 4 & 40.0 & \\
\hline & Total & 10 & 100.0 & 10 & 100.0 & \\
\hline
\end{tabular}




\section{DISCUSSION}

As SIE influenced significantly the bonding effectiveness to zirconia, null hypothesis (1) was rejected. Null hypothesis (2) was also rejected, as mechanical aging affect bonding effectiveness in TSS groups.

Results of this study emphasized superior MTBS results of SIE than TSS groups and were consistent with previous studies ${ }^{(17-21)}$. Fracture mode analysis for SIE groups remained primarily cohesive in the resin cement over the testing intervals (fig.2), while TSS groups showed an increase in the percentage of interfacial failure between zirconia and adhesive resin with water storage, indicating that the established bond was liable to hydrolysis and degradation in the presence of water ${ }^{(22)}$ (Fig. 4).

Procedure of selective infiltration etching create a retentive architecture (fig. 1) that differs from airborne particle abrasion as it is self-introduced material without applied external mechanical stresses, second, it occurs on ultrastructural grain level without creation of surface defects on material loss, and finally it creates 3-dimensional retentive features where the adhesive resin can infiltrate ${ }^{(23)}$.

Tribo-chemicalsilica sandblasting was opted in this study since this form of treatment -against conventional sandblasting using alumina powder -appeared as the most effective mechanical pre-treatment of zirconia regarding bonding receptiveness. Some authors ${ }^{(24,25)}$ reported that sandblasting may negatively influence the properties of zirconia ceramics, it can produce micro-cracks and thus decrease the strength and longevity of zirconia.

In vitro dental researches conventional methods for aging the specimens are storage in deionized water, thermocycling, fatigue strength testing and chemical degradation in $\mathrm{NaOH}$ solution ${ }^{(26,27)}$. In the current study, 4 weeks aging period was selected. Oczan et al. ${ }^{(28)}$, in a recent systematic review found that number of storage days varied enormously between 0.16 and 730 days, in different studies on bonding to ceramic oxide.
As the size of bonding area in the current study was relatively small; thus a faster aging effect could be expected. Data from 4 weeks immersion in this study showed a significant decrease in bond strength in TSS group. Degradation of resin -zirconia bonding might be attributed to water sorption, leading to hydrolysis degradation ${ }^{(29)}$. Water may decrease bond strength, especially to polycrystalline ceramics such as alumina and zirconia, as they can establish only weak chemical bonds to organophosphate based monomers as $10-\mathrm{MDP}^{(30)}$.

In this study, short term water storage might be considered as one of the limitations, because long term water storage will deteriorate the bond and affect the data to a greater extent ${ }^{(31)}$.

TSS was not effective in creating adequate retentive features (fig. 4), which explains the lower MTBS values observed. On the contrary, TSS abrasion resulted in the creation of non-retentive sharp cracks which may act as stress concentration sites that could further weaken zirconia-resin bond strength $^{(23)}$. Modified nanomechanical retention of SIE group was confirmed by the significantly higher bond strength of $45.9 \mathrm{MPa}$ (4 weeks), while only 24.9 MPa (4 week) was found for TSS specimens bonded with same adhesive resin. Fracture mode for SIE group remained primarily cohesive in resin cement over testing intervals, meanwhile TSS group showed interfacial failure.

Composite resin cement including 10-MDP (10 methacryloyloxydecyl dihydrogen phosphate) are the recommended cement for zirconia restorations, because the chemical interaction between the hydroxyl groups of oxide ceramic and organophosphate ester monomer of MDP in cement $^{(28)}$.

Selective infiltration etching (SIE) change the low surface energy surface of zirconia to a highly active and retentive surface. A systematic review searched for highest bond strength values of adhesion to zirconia and found surface conditioning and MDP monomer based cement showed the 
highest bond strength results (ranging from 17.1 to $42.3 \mathrm{MPa}$, depending on testing and aging method) ${ }^{(32)}$.

Microtensile bond strength testing has many advantages over other bond strength test methods as applied load is vertical to the bonded area and small specimen size reduces the percentage of incorporation of structural defects, resulting in less scattering of data ${ }^{(33)}$. Microtensile bond strength test is a meticulous method, which requires investing time and effort, especially during preparing and cutting the specimens, to avoid damage of microbars. When combined with water storage as a type of artificial aging process, not only accurate determination of the initial bond strength value is obtained, but also the long-term performance of this bond may be predicted ${ }^{(34)}$.

As the present data were obtained under controlled laboratory conditions, additional in vivo studies are needed to observe the long-term performance of the achieved bond strength. More investigations are required to optimize selective infiltration etching technique in terms of the resultant surface changes and technique simplicity and to optimize the properties of the used adhesive resin.

\section{CONCLUSIONS}

1- Selective infiltration etching provided superior micro-tensile bond strength results compared to tribo-chemical silica sandblasting.

2- Established bond strength by selective infiltration etching was not affected by artificial aging unlike tribo-chemical silica sandblasting that showed decreasing in bond strength values after 4 weeks water storage.

\section{CLINICAL IMPLICATIONS}

Heat induced maturation and selective infiltration etching produced a reliable and profound zirconia resin bond strength resistant to artificial aging.

\section{REFERENCES}

1. Piconi C, Maccauro G. Zirconia as a ceramic biomaterial. Biomaterials 1999;20: 1-25.

2. Chevalier J, Deville S, Munch E, Jullian R, Lair F. Critical effect of cubic phase on aging in $3 \mathrm{~mol} \%$ yttria-stabilized zirconia ceramics for hip replacement prosthesis. Biomaterials 2004; 25:5539-45.

3. Lankford J, Page RA, Rabenberg L. Deformation mechanisms in yttria-stabilized zirconia. J Mater Sci 1988; 23:4144-4156.

4. Deville S, Chevalier J, El Attaoui H. Atomic force microscopy study and qualitative analysis of martensite relief in zirconia. JAm Ceram Soc 2005; 88:1261-1267.

5. Deville S, Chevalier J, Dauvergne C,- Fantozzi G, Bartolome JF, Moya JS, et al. Microstructural investigation of the aging behavior of (3Y-TZP)-Al2O3 composites. J Am Ceram Soc 2005; 88:1273-1280.

6- Derand P, Derand T. Bond strength of luting cements to zirconium oxide ceramics. Int J Prosthodont 2000; 13:131135.

7- Blatz MB, Sadan A, Kern M. Resin-ceramic bonding: a review of the literature. J Prosthet Dent 2003; 89:268-274.

8- Ural C, Kulunk T, Kulunk S, Kurt M. The effect of laser treatment on bonding between zirconia ceramic surface and resin cement. Acta Odontol Scand 2010; 68:354-359.

9- Chen L, Suh B1, Kim J, Tay FR. Evaluation of silicacoating techniques for zirconia bonding. Am J Dent 2011; 24:79-84

10- Inokoshi M, Kameyama A, De Munck J, Minakuchi S, Van Meerbeek B. Durable bonding to mechanically and/ or chemically pre-treated dental zirconia. J Dent 2013; 41:170-179.

11. Inokoshi M, Poitevin A, De Munck J, Minakuchi S, Van Meerbeek B. Bonding effectiveness to different chemically pre-treated dental zirconia. Clin Oral Investig 2014;18: 1803-1812.

12- Kern M, Barloi A, Yang B (2009) Surface conditioning influences zirconia ceramic bonding. J Dent Res 88(9):817-822.

13- Derand T, Molin M, Kvam K (2005) Bond strength of composite luting cement to zirconia ceramic surfaces. Dent Mater 21(12):1158-1162 
14- Ozcan M, Nijhuis H, Valandro LF (2008) Effect of various surface conditioning methods on the adhesion of dual-cure resin cement with MDP functional monomer to zirconia after thermal aging. Dent Mater J 27(1):99-104.

15- Yoshida K, Tsuo Y, Meng X, AtsutaM (2007) Mechanical properties of dual-cured resin luting agents for ceramic restoration. J Prosthodont 16(5):370-376.

16- Samimi P, Hasankhani A, Matinlinna J, Mirmohammadi H (2015) Effect of Adhesive Resin Type for Bonding to Zirconia Using Two Surface Pretreatments. J Adhes Dent 17(1): 353-359.

17- El Zohairy AA, De Gee AJ, Mohsen MM, Feilzer AJ. Microtensile bond strength testing of luting cements to prefabricated CAD/CAM ceramic and composite blocks. Dent Mater 2003; 19:575-83.

18- Friederich R, Kern M. Resin bond strength to densely sintered alumina ceramic. Int J Prosthodont 2002; 15: 333338 .

19- de Souza G, Henning D, Aggarwal A, Tam LE. The use of MDP-based materials for bonding to zirconia .J Prosthet Dent 2014; 112:895-902.

20- Kamada K, Yoshida K, Atsuta M. Early bond strength and durability of bond between a ceramic material and chemically-cured or dual-cured resin luting agent. Am J Den 2001; 14:85-88.

21- Kern M. Resin bonding to oxide ceramics for dental restorations .J Adhes Sci Technol 2009;23: 1097-1011.

22- Wegner SM, Gerdes W, Kern M. Effect of different artificial aging conditions on ceramic-composite bond strength. Int J Prosthodont 2002;15: 267-72.

23- Amaral R, Ozcan M, Bottino MA, Valandro LF. Microtensile bond strength of a resin cement to glass infiltrated zirconia-reinforced ceramic: the effect of surface conditioning. Dent Mater 2006;22: 283-90.
24. Kosmac T, Oblak C, Jevnikar P, Funduk N,Marion L (2000) Strength and reliability of surface treated Y-TZP dental ceramics. J Biomed Mater Res 53(4):304-313

25. Zhang Y, Lawn BR, Rekow ED, Thompson VP (2004) Effect of sandblasting on the long-term performance of dental ceramics. J Biomed Mater Res B 71(2):381-386.

26- Hummel M, Kern M. Durability of the resin bond strength to the alumina ceramic Procera. Dent Mater 2004;20:498508 .

27- Luthy H, Loeffel 0, Hammerle CH. Effect of thermocycling on bond strength of luting cements to zirconia ceramic Dent Mater 2006;22:195-200.

28- Ozcan M, Bernasconi M. Adhesion to zirconia used for dental restorations:a systematic review and meta-analysis. J Adhes Dent 2015;17:7-26.

29- Wolfart M, Lehmann F, Wolfart S, Kern M. Durability of the resin bond strength to zirconia ceramic after using different surface conditioning methods. Dent Mater 2007; 23:45-50..

30- Arikawa H, Kuwahata H, Seki H, Kanie T, Fuji K, Inoue $\mathrm{K}$. Deterioration of mechanical properties of composite resins. Dent Mater J 1995;14:78-83.

31- Soderholm K-JM, Roberts MJ. Influence of water exposure on the tensile strength of composites. J Dent Res 1990;69:1812-1816.

32- Kern M. Resin bonding to oxide ceramics for dental restorations. J Adhes Sci Technol 2009;23:1097-1111.

33- Aboushelib MN, Kleverlaan CJ, Feilzer AJ.Microtensile bond strength of different components of core veneered allceramic restorations: Part II: Zirconia veneering ceramics. Dent Mater 2006;22:857-63.

34- Blatz MB, Sadan A, Kern M. Resin-ceramic bonding: A review of the literature. J Prosthet Dent 2003;89:268-74. 